\title{
Qualidade de produtos comerciais à base de frutas
}

\section{Quality assessment of commercial fruit products}

\author{
Diego Gadelha Souza $a^{1}$,Jaqueline de Sousa Gomes ${ }^{2}$, Amanda Kelly da Silva², Deocleciano Cassiano de \\ Santana Neto ${ }^{4}$, Flávia de Brito Martins ${ }^{5}$, Adriana Ferreira dos Santos ${ }^{6}$
}

${ }^{1}$ Mestrando em Engenharia de Alimentos, Universidade Federal do Paraná, Curitiba, +554198716-6604, diegogs93@gmail.com; ${ }^{2}$ Mestranda em Sistemas Agroindustriais, Universidade Federal de Campina Grande, Pombal, jaquelinesousa_pb@hotmail.com; ${ }^{3}$ Engenheira de Alimentos, Universidade Federal de Campina Grande, amanda_dkelly@hotmail.com; ${ }^{4}$ Doutorando em Engenharia de Alimentos, Universidade Federal do Paraná, Curitiba, deocleciano.cassiano7@gmail.com; ${ }^{5}$ Engenheira de Alimentos, Universidade Federal de Campina Grande, flaviabrito01@gmail.com; ${ }^{6}$ Professora do Centro de Ciências e Tecnologia Agroalimentar, Universidade Federal de Campina Grande, Pombal, adrefesantos@yahoo.com.br.

\section{A R T I G O \\ Recebido: $26 / 08 / 2019$ \\ Aprovado: 07/02/2020}

\section{Palavras-chave:}

Doce em massa

Polpa

Compostos bioativos

Key words:

Sweet pastry

Pulp

Quality

\section{R E S U M O}

$\mathrm{O}$ processamento de frutos in natura em produtos derivados proporcionam a conservação e o consumo fora do período de safra, no entanto, é importante que estes produtos cheguem ao mercado consumidor atendendo e garantindo a sua qualidade físico-química e funcional. Desta forma, o objetivo deste trabalho foi avaliar características físico-químicas e quantificação de compostos bioativos em amostras comerciais de doces em massa de goiaba e banana, polpas de acerola e goiaba. Os produtos adquiridos foram representados por quatro marcas comerciais, provenientes dos mercados locais de Pombal e Patos na Paraíba. Foram realizadas as determinações de sólidos solúveis, acidez titulável, $\mathrm{pH}$, ratio, teor de água, resíduo mineral fixo, ácido ascórbico, clorofila, carotenoides, flavonoides, antocianinas e polifenóis extraíveis totais. Para o teor de cinzas, a amostra A de doce de goiaba se destacou entre as demais, indicando maior quantidade de resíduo inorgânico. As amostras A, B e C de doce de goiaba e amostra D de doce de banana apresentaram os maiores teores de compostos fenólicos. A amostra $\mathrm{C}$ de doce de banana apresentou o teor mais elevado de sólidos solúveis. A amostra D de polpa de acerola obteve o melhor resultado para ácido ascórbico e compostos fenólicos. Para o $\mathrm{pH}$, somente as amostras B, C e D de polpa de goiaba encontram-se dentro do permitido pela legislação brasileira.

\section{A B S T R A C T}

The processing of fresh fruits into derived products provides conservation and consumption outside the harvest period, however, it is important that these products reach the consumer market, attending to and guaranteeing their physical-chemical and functional quality. Thus, the objective of this work was to evaluate physicochemical characteristics and quantification of bioactive compounds in commercial samples of guava and banana sweets, and acerola and guava pulps. The products purchased were represented by four commercial brands, coming from the local markets of Pombal and Patos in Paraíba. Determinations of soluble solids, titratable acidity, $\mathrm{pH}$, ratio, water content, fixed mineral residue, ascorbic acid, chlorophyll, carotenoids, flavonoids, anthocyanins and total extractable polyphenols were carried out. For ash content, sample A of guava jam stood out among the others, indicating a greater amount of inorganic residue. Samples A, B and C of guava jam and sample D of banana jam had the highest content of compounds phenolic. The sweet banana sample $\mathrm{C}$ showed the highest content of soluble solids. Acerola pulp sample D obtained the best result for ascorbic acid and phenolic compounds. For $\mathrm{pH}$, only samples $\mathrm{B}, \mathrm{C}$ and $\mathrm{D}$ of guava pulp are within the limits allowed by Brazilian legislation.

\section{INTRODUÇÃO}

Os frutos fornecem componentes importantes ao nosso organismo, como por exemplo, ácido ascórbico, carotenoides, antocianinas, flavonoides, fontes de compostos bioativos que atuam em funções básicas, ligados à prevenção de doenças por meio da ação antioxidante, substâncias capazes de inibir a oxidação, reduzindo a concentração de radicais livres no organismo (MORAIS et al., 2017; VIANA et al., 2015).

No entanto, os frutos são alimentos perecíveis, por isso, para reduzir os processos de degradação é preciso obter algumas condições de conservação, transformando os frutos

\section{Revista Verde}

ISSN 1981-8203

Pombal, Paraíba, Brasil v. 15, n.2, abr.-jun., p.146-152, 2020

doi: $10.18378 /$ rvads.v15i2.6944 
in natura em produtos processados como doces, polpas, sucos e extratos, por exemplo. Porém, deve-se levar em consideração que algumas técnicas podem comprometer a atividade dos compostos encontrados nesses frutos, bem como alterar suas características físico-químicas (MORAIS et al., 2017).

Além disso, os consumidores têm se tornado cada vez mais exigente e criterioso com a qualidade dos produtos que consomem. É crescente a preocupação em fazer uso de produtos o mais próximo possível da naturalidade, para evitar o consumo de substâncias sintetizadas quimicamente e aumentar a ingestão de compostos bioativos (SILVA, 2014).

É importante também que as indústrias alimentícias se adequem aos padrões exigidos pela legislação vigente e voltem para o aumento da composição funcional de seus produtos, que além de nutrir exercem outras funções no organismo.

Desta forma, o objetivo deste trabalho foi avaliar características físico-químicas e a quantificação de compostos bioativos em amostras comerciais de doce em massa de goiaba e banana, amostras de polpa de frutas de acerola e goiaba.

\section{MATERIAL E MÉTODOS}

O experimento foi desenvolvido no Centro de Ciências e Tecnologia Agroalimentar (CCTA), Unidade Acadêmica de Tecnologia de Alimentos (UATA) da Universidade Federal de Campina Grande (UFCG), no Laboratório de Tecnologia de Produtos de Origem Vegetal (LTPOV) em Pombal, Paraíba.

Foram adquiridos produtos comerciais de 4 marcas, nos mercados locais de Pombal-PB e de Patos-PB. Dentre os produtos comerciais foram avaliados, doces em massa de goiaba e banana, polpas de acerola e goiaba. As marcas para cada produto comercial foram representadas por Amostras A, B, C e D, ou seja, para o doce em massa de goiaba foram avaliadas 4 amostras, e respetivamente para os demais produtos em estudo.

Após a aquisição dos produtos, estes foram transportados para o LTPOV/UATA/CCTA, em Pombal-PB para as avaliações físico-químicas e de compostos bioativos.

\section{Avaliação Físico-Química}

Acidez Titulável: AT (\% de ácido cítrico): por titulometria com $\mathrm{NaOH} \mathrm{0,1} \mathrm{M,} \mathrm{segundo} \mathrm{Instituto} \mathrm{Adolfo} \mathrm{Lutz}$ - IAL (2008).

pH: determinado em pHmetro, com inserção direta do eletrodo, de acordo com IAL (2008).

Sólidos solúveis $(\%)$ : por refratômetro digital, segundo Association of Official Analytical Chemists AOAC (2005);

Ratio: obtido pelo quociente dos valores de sólidos solúveis e acidez titulável;

Teor de água (\%): determinada por meio de secagem em estufa a $105{ }^{\circ} \mathrm{C}$ até peso constante de acordo com método do Instituto Adolfo Lutz (IAL, 2008);

Cinzas (\%): determinada pela incineração da amostra em mufla a $550{ }^{\circ} \mathrm{C}$ até as cinzas ficarem brancas ou ligeiramente acinzentadas (IAL, 2008).

\section{Avaliação Compostos Bioativos}

Ácido ascórbico $\left(\mathrm{mg} .100 \mathrm{~g}^{-1}\right)$ : quantificado utilizando-se método de titulometria com solução de DFI (2,6 diclorofenolindofenol a $0,2 \%$ ), até coloração rósea clara permanente, segundo AOAC (2005);

Carotenoides Totais $\left(\mu \mathrm{g} . \mathrm{g}^{-1}\right)$ : determinados com cerca de $1 \mathrm{~g}$ de amostra, macerada com $0,2 \mathrm{~g}$ de carbonato de cálcio $\left(\mathrm{CaCO}_{3}\right)$ e $5 \mathrm{~mL}$ de acetona $(80 \%)$ gelada, em ambiente escuro. Em seguida, as amostras foram centrifugadas a $10{ }^{\circ} \mathrm{C}$ e $3.000 \mathrm{rpm}$ por $10 \mathrm{~min}$ e os sobrenadantes foram lidos em espectrofotômetro nos comprimentos de onda de 470, 646 e $663 \mathrm{~nm}$, de acordo com Lichtenthaler (1987);

Flavonoides e antocianinas (mg.100g-1): determinados com cerca de $1 \mathrm{~g}$ de amostra, macerada com $10 \mathrm{~mL}$ de solução extratora de etanol $95 \%$ mais $\mathrm{HCl} 1,5 \mathrm{~N}$ na proporção 85:15 (v/v). Em seguida, transferidas para tubos (envoltos com papel alumínio) e deixados em repouso por $24 \mathrm{~h}$ sob refrigeração. As amostras foram filtradas em papel de filtro e as leituras foram realizadas em espectrofotômetro a $374 \mathrm{~nm}$ para os flavonoides e $535 \mathrm{~nm}$ para as antocianinas, as determinações seguiram a metodologia de Francis (1982);

Polifenóis extraíveis totais (mg.GAE. $100 \mathrm{~g}^{-1}$ ): determinados pesando aproximadamente $1 \mathrm{~g}$ das amostras, diluídas em água e acrescidas de $0,125 \mathrm{~mL}$ do reagente FolinCiocalteau, seguido de agitação e repouso por 5 min. Foram utilizadas alíquotas distintas para os produtos e amostras analisadas. Logo após o tempo de reação, foram adicionados $0,250 \mathrm{~mL}$ de carbonato de sódio $20 \%$, seguida de nova agitação e repouso em banho-maria a $40{ }^{\circ} \mathrm{C}$ por $30 \mathrm{~min}$. A curva padrão foi preparada com ácido gálico e as leituras foram realizadas em espectrofotômetro a $765 \mathrm{~nm}$, estimados de acordo com o método de Folin-Ciocalteau descrito por Waterhouse (2006).

\section{Delineamento Experimental e Análise Estatística}

O experimento foi instalado segundo um delineamento inteiramente casualizado com 2 produtos comerciais (doce em massa, polpa de frutas). Os doces em massa e as polpas foram representadas por 2 sabores de frutos, 4 amostras e 4 repetições cada. Os dados obtidos foram submetidos à análise de variância pelo teste $\mathrm{F}$ e as médias foram comparadas pelo teste de Tukey em nível de $5 \%$ de probabilidade, utilizando o programa computacional ASSISTAT versão 7.7 (SILVA; AZEVEDO, 2016).

\section{RESULTADOS E DISCUSSÃO}

Os doces em massa de goiaba analisados no presente estudo não possuem legislação específica com Padrões de Identidade e Qualidade (PIQs) fixadas pelo Ministério da Agricultura e do Abastecimento (MAPA). A Resolução Normativa n. 9 de 1978, estabelece apenas o valor mínimo de $65 \%$ para teor de sólidos solúveis para doce em massa, desta forma, as amostras avaliadas (Tabela 1) encontram-se nos padrões estabelecidos por essa legislação para este parâmetro (BRASIL, 1978). Vale ressaltar, que a concentração abaixo de $65 \%$ pode causar pouca ou nenhuma inversão da sacarose e incompleta absorção do açúcar pelo fruto, podendo afetar a conservação do produto.

Quanto ao $\mathrm{pH}$, as amostras obtiveram valores abaixo de 4,5, produtos abaixo desse valor, apresentam uma maior estabilidade quanto ao desenvolvimento de microrganismos, limitando o crescimento de leveduras, fungos e bactérias sensíveis (MARTINS et al., 2017). 
Tabela 1. Avaliação físico-química de quatro amostras comerciais de doce em massa de goiaba.

\begin{tabular}{ccccccc}
\hline Amostras & SS (\%) & $\mathrm{pH}$ & $\begin{array}{c}\text { Acidez Titulável } \\
(\% \text { ác. Cítrico) }\end{array}$ & Ratio & $\begin{array}{c}\text { Teor } \\
\text { de água }(\%)\end{array}$ & Cinzas $(\%)$ \\
\hline A & $75,3 \pm 0,99 \mathrm{a}^{*}$ & $3,68 \pm 0,10 \mathrm{~b}$ & $0,67 \pm 0,14 \mathrm{a}$ & $123,4 \pm 10,29 \mathrm{a}$ & $30,33 \pm 2,26 \mathrm{~b}$ & $0,63 \pm 0,02 \mathrm{a}$ \\
$\mathrm{B}$ & $73,8 \pm 0,92 \mathrm{a}$ & $3,88 \pm 0,92 \mathrm{a}$ & $0,73 \pm 0,12 \mathrm{a}$ & $108,8 \pm 12,53 \mathrm{ab}$ & $37,55 \pm 1,09 \mathrm{a}$ & $0,55 \pm 0,05 \mathrm{~b}$ \\
$\mathrm{C}$ & $74,4 \pm 1,04 \mathrm{a}$ & $3,57 \pm 0,06 \mathrm{~b}$ & $0,64 \pm 0,03 \mathrm{a}$ & $115,6 \pm 5,58 \mathrm{ab}$ & $32,44 \pm 0,34 \mathrm{~b}$ & $0,55 \pm 0,02 \mathrm{~b}$ \\
$\mathrm{D}$ & $73,7 \pm 0,54 \mathrm{a}$ & $3,85 \pm 0,05 \mathrm{a}$ & $0,73 \pm 0,06 \mathrm{a}$ & $101,3 \pm 7,87 \mathrm{~b}$ & $38,36 \pm 2,00 \mathrm{a}$ & $0,47 \pm 0,03 \mathrm{c}$ \\
\hline
\end{tabular}

*Médias seguidas por uma mesma letra, na mesma coluna, não diferem significativamente entre si pelo teste de Tukey ( $\mathrm{p} \leq 0,05)$.

As amostras apresentaram baixa acidez, esses resultados são esperados, pois os frutos utilizados na composição de doce encontram-se em estado de maturação avançado, nesse período os mesmos utilizam os ácidos orgânicos como substratos em seu metabolismo (FONSECA, 2012).

Lima et al., (2015) afirmaram que o ratio, indica o grau de doçura de um fruto ou de seu produto, evidenciando qual o sabor predominante, o doce ou o ácido, ou ainda se há equilíbrio entre eles. Os resultados das amostras de doce de goiaba mostram que os produtos apresentaram uma boa palatabilidade, por demonstrar um equilíbrio satisfatório entre o teor de sólidos solúveis e ácidos.
Com relação aos resultados obtidos para o teor de água dos doces de goiaba, estes apresentaram baixos teores, demonstrando uma maior estabilidade, qualidade, eficiência do cozimento, ou seja, menor sensibilidade à deterioração.

Para o teor de cinzas, a amostra A se destacou entre as demais, indicando maior quantidade de resíduo inorgânico, que é constituída principalmente de minerais. É possível que o grau de refinamento da polpa utilizada, bem como a pureza do açúcar utilizada na formulação dos doces, influencie no teor final de cinzas no produto.

A Tabela 2 apresenta os resultados de compostos bioativos de quatro amostras comerciais de doce em massa de goiaba.

Tabela 2. Compostos Bioativos de quatro amostras comerciais de doce em massa de goiaba.

\begin{tabular}{|c|c|c|c|c|c|}
\hline Amostras & $\begin{array}{c}\text { Ácido } \\
\text { Ascórbico } \\
\left(\mathrm{mg}^{1} 100 \mathrm{~g}^{-1}\right)\end{array}$ & $\begin{array}{l}\text { Carotenoides } \\
\left(\mu \mathrm{g} .100 \mathrm{~g}^{-1}\right)\end{array}$ & $\begin{array}{l}\text { Antocianinas } \\
\left(\mathrm{mg} .100 \mathrm{~g}^{-1}\right)\end{array}$ & $\begin{array}{l}\text { Flavonoides } \\
\left(\mathrm{mg} 100 \mathrm{~g}^{-1}\right)\end{array}$ & $\begin{array}{c}\text { Compostos fenólicos } \\
\text { (mg. } 100 \mathrm{~g}^{-1} \text { de ácido gálico) }\end{array}$ \\
\hline $\mathrm{A}$ & $16,42 \pm 1,29 b^{*}$ & $6,58 \pm 0,76 a b$ & $0,38 \pm 0,34 \mathrm{a}$ & $2,63 \pm 1,31 \mathrm{a}$ & $188,01 \pm 4,04 \mathrm{a}$ \\
\hline $\mathrm{B}$ & $24,35 \pm 3,08 \mathrm{a}$ & $6,22 \pm 0,42 b$ & $0,23 \pm 0,15 a$ & $4,10 \pm 0,37 \mathrm{a}$ & $154,24 \pm 2,40 b$ \\
\hline $\mathrm{C}$ & $14,28 \pm 1,39 b$ & $6,96 \pm 0,48 a b$ & $0,31 \pm 0,12 \mathrm{a}$ & $2,88 \pm 0,93 \mathrm{a}$ & $136,77 \pm 1,66 \mathrm{c}$ \\
\hline $\mathrm{D}$ & $9,74 \pm 0,87 \mathrm{c}$ & $7,73 \pm 0,63 \mathrm{a}$ & $0,16 \pm 0,05 a$ & $3,47 \pm 0,39 a$ & $77,91 \pm 2,04 \mathrm{~d}$ \\
\hline
\end{tabular}

*Médias seguidas por uma mesma letra, na mesma coluna, não diferem significativamente entre si pelo teste de Tukey $(\mathrm{p} \leq 0,05)$.

Quanto ao teor de ácido ascórbico, os resultados disponíveis na literatura demonstram grandes variações em função do tipo de produto e das condições nas quais foram processados. No entanto, todos destacam que o ácido ascórbico é muito suscetível à degradação por diversos fatores, principalmente pela temperatura. Como parte do processamento, doces em massa passam por processo de cocção e ainda são armazenados em temperatura ambiente, favorecendo a degradação desta vitamina (FREDA, 2014).

A recomendação diária desse composto para atender as necessidades biológicas é relativo a idade do indivíduo. Os mínimos recomendados estão correlacionados de acordo com a legislação, onde para adultos (90 mg), crianças $(60-70 \mathrm{mg})$ e gestantes (110 mg) (BRASIL, 2005). Dessa forma, todas as amostras analisadas de doces em massa de goiaba em estudo não atende a quantidade diária estabelecida, devendo-se consumir uma grande quantidade para satisfazer a necessidade, não sendo ideal, devido ao alto teor de açúcar.

O teor de carotenoides apresentaram baixos teores para as amostras de doce em massa de goiaba, quando comparado com a literatura. Freda et al., (2018) avaliando compostos bioativos em doce de goiaba obtiveram teor médio de 50,10 $\mu \mathrm{g} . \mathrm{g}^{-1}$ para doce convencional, superior ao do presente estudo.

Assim como para carotenoides os teores médios obtidos para antocianinas e flavonoides nas amostras analisadas foram baixos. No entanto, vale salientar, que a composição química dos frutos é influenciada por fatores, como cultivar, estádio de maturação, clima, condições de cultivo, processamento e armazenamento (HAIDA et al., 2015). Além disso, durante o processamento, as goiabas são submetidas a um aquecimento elevado, na qual ocorrem grandes perdas desses compostos, reduzindo o teor no produto final.

Os fatores citados anteriormente também contribuem consideravelmente para o teor de compostos fenólicos, observa-se que houve diferenças significativas entre as amostras avaliadas, com a amostra A apresentando o maior teor. Os resultados avaliados foram inferiores ao observados por Freda et al., (2018) que obtiveram $649,19 \mathrm{mg} 100 \mathrm{~g}^{-1} \mathrm{de}$ ácido gálico em doce de goiaba.

Os resultados obtidos podem contribuir para a fixação dos PIQ's dos doces em massa de goiaba avaliados, além disso, não fornecer informações normativas para produtos firmados comercialmente e reconhecidos pelos consumidores há décadas acarreta uma abertura de brechas para a falta de padronização nas indústrias alimentícias (OLIVEIRA et al., 2018).

$\mathrm{Na}$ Tabela 3 encontram-se os valores médios para as avaliações físico-químicas do doce em massa de banana.

Como citado anteriormente, o valor mínimo de sólidos solúveis para doce em massa estabelecido pela legislação é de 65\% (BRASIL, 1978). Desta forma, as amostras avaliadas encontram-se nos padrões estabelecidos por essa legislação para este parâmetro, com a amostra A apresentando o teor mais elevado. 
Tabela 3. Avaliação físico-química de quatro amostras comerciais de doce em massa de banana.

\begin{tabular}{ccccccc}
\hline \multirow{2}{*}{ Amostras } & \multirow{2}{*}{ SS $(\%)$} & $\mathrm{pH}$ & $\begin{array}{c}\text { Acidez Titulável } \\
(\% \text { ác. Cítrico) }\end{array}$ & Ratio & $\begin{array}{c}\text { Teor de água } \\
(\%)\end{array}$ & Cinzas $(\%)$ \\
\hline A & $77,2 \pm 0,45 \mathrm{a}^{*}$ & $4,08 \pm 0,06 \mathrm{~b}$ & $0,59 \pm 0,03 \mathrm{~b}$ & $127,6 \pm 0,78 \mathrm{~b}$ & $31,95 \pm 1,55 \mathrm{c}$ & $0,52 \pm 0,03 \mathrm{~b}$ \\
$\mathrm{~B}$ & $67,0 \pm 0,65 \mathrm{~d}$ & $4,47 \pm 0,01 \mathrm{a}$ & $0,59 \pm 0,06 \mathrm{~b}$ & $109,5 \pm 5,75 \mathrm{c}$ & $38,40 \pm 1,16 \mathrm{a}$ & $0,73 \pm 0,04 \mathrm{a}$ \\
$\mathrm{C}$ & $76,2 \pm 0,05 \mathrm{~b}$ & $3,88 \pm 0,06 \mathrm{c}$ & $0,49 \pm 0,02 \mathrm{c}$ & $159,2 \pm 4,59 \mathrm{a}$ & $34,89 \pm 1,16 \mathrm{bc}$ & $0,52 \pm 0,04 \mathrm{~b}$ \\
$\mathrm{D}$ & $68,4 \pm 0,50 \mathrm{c}$ & $4,08 \pm 0,14 \mathrm{~b}$ & $0,70 \pm 0,01 \mathrm{a}$ & $98,0 \pm 1,69 \mathrm{~d}$ & $37,67 \pm 2,25 \mathrm{ab}$ & $0,68 \pm 0,07 \mathrm{a}$ \\
\hline
\end{tabular}

*Médias seguidas por uma mesma letra, na mesma coluna, não diferem significativamente entre si pelo teste de Tukey (p $\leq 0,05)$.

Segundo Bezerra et al., (2019), a medida de pH de um alimento deve ser considerada na sua avaliação da qualidade, sob o ponto de vista microbiológico e químico. $\mathrm{Na}$ avaliação microbiológica, os alimentos se dividem em $\mathrm{pH}$ inferiores ou superiores a 4,5 que, de modo geral, indicam o seu grau de deterioração, atestado pela acidez ou basicidade desenvolvida. Nos aspectos químicos, a maior parte das reações ocorre durante o armazenamento e processamento dos alimentos e são significativamente alteradas, devido à variação da concentração hidrogeniônica do meio (GOMES; OLIVEIRA, 2011). Quanto ao teor de $\mathrm{pH}$, todas as amostras apresentaram valores inferiores a 4,5 e superior ao obtido por Kato et al., (2013) avaliando doce de frutas, com teor médio de 3,78.

A determinação da acidez em conjunto com a medida de pH são parâmetros que pode contribuir na avaliação da qualidade tecnológica, nutricional e microbiológica destes produtos (BEZERRA et al., 2019). Todos os produtos apresentaram baixa acidez, com valores inferiores ao obtido por Kato et al., (2013) avaliando doce de frutas, com teor médio de $9,06 \%$.

Quanto ao ratio, as amostras de doce em massa de banana apresentaram uma boa palatabilidade, por demonstrar um equilíbrio satisfatório entre o teor de sólidos solúveis e ácidos. A amostra $\mathrm{C}$ apresentou o teor mais elevado, devido a elevada presença de sólidos solúveis e baixa acidez.

Com relação aos resultados obtidos para o teor de água dos doces de banana, estes apresentaram baixos teores, demonstrando uma maior estabilidade, por ter uma menor porcentagem, com maior destaque para a amostra A com teor médio de 31, 95\%. Para o teor de cinzas, a amostra B se destacou entre as demais, indicando maior quantidade de resíduo inorgânico, que é constituída principalmente de minerais.

Na Tabela 4 encontram-se os resultados para compostos bioativos de doce em massa de banana.

Tabela 4. Compostos Bioativos de quatro amostras comerciais de doce em massa de banana.

\begin{tabular}{|c|c|c|c|c|c|}
\hline Amostras & $\begin{array}{l}\text { Ácido Ascórbico } \\
\left(\mathrm{mg} .100 \mathrm{~g}^{-1}\right)\end{array}$ & $\begin{array}{l}\text { Carotenoides } \\
\left(\mu \mathrm{g} .100 \mathrm{~g}^{-1}\right)\end{array}$ & $\begin{array}{l}\text { Antocianinas } \\
\left(\mathrm{mg} .100 \mathrm{~g}^{-1}\right)\end{array}$ & $\begin{array}{l}\text { Flavonoides } \\
\left(\mathrm{mg} .100 \mathrm{~g}^{-1}\right)\end{array}$ & $\begin{array}{c}\text { Compostos fenólicos } \\
\text { (mg.100 } \mathrm{g}^{-1} \text { de ácido gálico) }\end{array}$ \\
\hline $\mathrm{A}$ & $3,76 \pm 0,57 \mathrm{ab}^{*}$ & $1,20 \pm 0,26 a$ & $0,10 \pm 0,11 \mathrm{a}$ & $1,36 \pm 0,13 \mathrm{ab}$ & $58,70 \pm 1,39 \mathrm{c}$ \\
\hline $\mathrm{B}$ & $4,57 \pm 0,0 \mathrm{a}$ & $0,76 \pm 0,25 a b$ & $0,08 \pm 0,07 \mathrm{a}$ & $2,18 \pm 0,28 \mathrm{a}$ & $87,10 \pm 1,34 \mathrm{~b}$ \\
\hline $\mathrm{C}$ & $3,31 \pm 0,58 b$ & $0,57 \pm 0,21 b$ & $0,03 \pm 0,03 a$ & $0,80 \pm 0,33 b$ & $50,33 \pm 0,84 \mathrm{~d}$ \\
\hline $\mathrm{D}$ & $4,57 \pm 0,01 \mathrm{a}$ & $0,67 \pm 0,09 \mathrm{~b}$ & $0,24 \pm 0,33 \mathrm{a}$ & $1,08 \pm 0,71 \mathrm{~b}$ & $146,45 \pm 1,01 \mathrm{a}$ \\
\hline
\end{tabular}

*Médias seguidas por uma mesma letra, na mesma coluna, não diferem significativamente entre si pelo teste de Tukey $(\mathrm{p} \leq 0,05)$.

Quanto ao teor de ácido ascórbico, observou-se um teor mais elevado para as amostras B e D, quando comparada às demais, entretanto, abaixo dos valores estimados para banana in natura, provavelmente essa redução pode ser decorrente do tratamento térmico em que estes produtos foram submetidos.

A recomendação diária de ácido ascórbico para atender as necessidades biológicas de acordo com a legislação para adultos são de $90 \mathrm{mg}$, crianças de 60-70 mg e gestantes de $110 \mathrm{mg}$ (BRASIL, 2005). Dessa forma, todas as amostras analisadas de doces em massa de banana em estudo não atende a quantidade diária estabelecida.

Os teores médios obtidos para carotenoides, antocianinas e flavonoides nas amostras analisadas foram relativamente baixos. No entanto, vale salientar, que os fatores ambientais, influenciam consideravelmente na composição química dos princípios alimentícios vegetais, bem como, o grau de maturação antes da colheita e as condições de maturação pós-colheita e de armazenagem (OLIVEIRA et al., 2019).
Observa-se que as amostras de doce em massa de banana apresentam-se como fontes significativas de compostos fenólicos, potencial benéfico à saúde, com destaque para a amostra $\mathrm{D}$, o que pode contribuir para sua conservação, uma vez que a atividade antioxidante desempenhada pelos fenólicos totais auxilia no aumento da vida útil do alimento.

Na Tabela 5, encontram-se os resultados obtidos para as avaliações físico-químicas em polpa de acerola. Para os sólidos solúveis a legislação exige o valor mínimo de $6 \%$ (BRASIL, 2018). Desta forma, somente as amostras A, B e D encontram-se dentro dos padrões. Os resultados para $\mathrm{pH}$ encontram-se dentro do permitido pela legislação brasileira, na qual exige valor mínimo de 2,80 para polpa de frutas (BRASIL, 2018). Além disso, todas as amostras obtiveram valores abaixo de 4,5, permitindo uma maior estabilidade quanto ao crescimento de microrganismos.

Tabela 5. Avaliação físico-química em polpa de acerola de quatro amostras comerciais.

\begin{tabular}{ccccccc}
\hline \multirow{2}{*}{ Amostras } & \multirow{2}{*}{$\mathrm{SS}(\%)$} & $\mathrm{pH}$ & $\begin{array}{c}\text { Acidez Titulável } \\
(\% \text { ác. Cítrico) }\end{array}$ & Ratio & $\begin{array}{c}\text { Teor de água } \\
(\%)\end{array}$ & Cinzas $(\%)$ \\
\hline $\mathrm{A}$ & $6,8 \pm 0,05 \mathrm{c}^{*}$ & $3,48 \pm 0,09 \mathrm{a}$ & $1,09 \pm 0,02 \mathrm{~b}$ & $6,2 \pm 0,14 \mathrm{~b}$ & $93,81 \pm 0,14 \mathrm{~b}$ & $0,26 \pm 0,07 \mathrm{~b}$ \\
$\mathrm{~B}$ & $7,8 \pm 0,00 \mathrm{a}$ & $3,45 \pm 0,05 \mathrm{a}$ & $1,06 \pm 0,02 \mathrm{~b}$ & $7,4 \pm 0,11 \mathrm{a}$ & $93,06 \pm 0,32 \mathrm{c}$ & $0,27 \pm 0,02 \mathrm{ab}$ \\
$\mathrm{C}$ & $4,3 \pm 0,08 \mathrm{~d}$ & $3,29 \pm 0,12 \mathrm{ab}$ & $1,09 \pm 0,01 \mathrm{~b}$ & $4,0 \pm 0,09 \mathrm{~d}$ & $96,12 \pm 0,02 \mathrm{a}$ & $0,16 \pm 0,02 \mathrm{c}$ \\
$\mathrm{D}$ & $7,2 \pm 0,08 \mathrm{~b}$ & $3,16 \pm 0,12 \mathrm{~b}$ & $1,34 \pm 0,03 \mathrm{a}$ & $5,4 \pm 0,13 \mathrm{c}$ & $92,93 \pm 0,35 \mathrm{c}$ & $0,34 \pm 0,03 \mathrm{a}$ \\
\hline
\end{tabular}

*Médias seguidas por uma mesma letra, na mesma coluna, não diferem significativamente entre si pelo teste de Tukey (p $\leq 0,05)$. 
De acordo com os resultados para acidez, as polpas de acerola, caracterizam-se como acidas, com a amostra D em maior percentual. As quatro amostras avaliadas apresentaram acidez em concordância com a legislação que estabelece valor mínimo 0,8\% (BRASIL, 2018). E superior ao reportado por Batista et al., (2013), que avaliando polpa de acerola obteve 0,69\% de ácido cítrico.

Para o ratio a amostra B apresentou o melhor equilíbrio entre sólidos solúveis e ácidos. Todas as amostras apresentaram palatabilidade superior a reportado por Adriano et al., (2011) avaliando a qualidade da polpa da acerola $(2,41)$.

Como esperado, todas as amostras obtiveram um elevado teor de água com maior destaque para a amostra $\mathrm{C}$, sendo necessário o uso de tecnologia simples, como o congelamento, para manter por maior tempo os nutrientes e estabilidade quanto ao crescimento de microrganismos. Para o teor de cinzas, a amostra D se destacou entre as demais, indicando maior quantidade de minerais.

Na Tabela 6 estão expressos os valores para compostos bioativos em polpas de acerola. Para ácido ascórbico há uma recomendação mínima de $800,0 \quad \mathrm{mg} .100 \mathrm{~g}^{-1}$ segundo a legislação para polpa de acerola (BRASIL, 2018). Com os resultados obtidos, a amostra $\mathrm{C}$, apresentou-se abaixo do permitido. A amostra D obteve o melhor resultado entre as amostras analisadas com 1492,63 mg.100 $\mathrm{g}^{-1}$.

Tabela 6. Avaliação de compostos bioativos em polpa de acerola de quatro amostras comerciais.

\begin{tabular}{cccccc}
\hline Amostras & $\begin{array}{c}\text { Ácido Ascórbico } \\
\left(\mathrm{mg} .100 \mathrm{~g}^{-1}\right)\end{array}$ & $\begin{array}{c}\text { Carotenoides } \\
\left(\mu \mathrm{g} .100 \mathrm{~g}^{-1}\right)\end{array}$ & $\begin{array}{c}\text { Antocianinas } \\
\left(\mathrm{mg} .100 \mathrm{~g}^{-1}\right)\end{array}$ & $\begin{array}{c}\text { Flavonoides } \\
\left(\mathrm{mg}_{\left.100 \mathrm{~g}^{-1}\right)}\right.\end{array}$ & $\begin{array}{c}\text { Compostos fenólicos } \\
\left(\mathrm{mg}^{100 \mathrm{~g}^{-1}} \mathrm{de} \text { ácido }\right. \\
\mathrm{gálico})\end{array}$ \\
\hline A & $976,81 \pm 2,44 \mathrm{c}^{*}$ & $5,84 \pm 0,15 \mathrm{a}$ & $7,22 \pm 0,21 \mathrm{~b}$ & $6,88 \pm 0,17 \mathrm{~b}$ & $815,97 \pm 39,25 \mathrm{c}$ \\
$\mathrm{B}$ & $1.104,07 \pm 5,09 \mathrm{~b}$ & $3,02 \pm 0,22 \mathrm{c}$ & $5,78 \pm 0,44 \mathrm{c}$ & $4,83 \pm 0,32 \mathrm{c}$ & $932,72 \pm 53,01 \mathrm{~b}$ \\
C & $568,54 \pm 2,50 \mathrm{~d}$ & $3,94 \pm 0,05 \mathrm{~b}$ & $3,43 \pm 0,14 \mathrm{~d}$ & $3,73 \pm 0,46 \mathrm{~d}$ & $395,98 \pm 21,39 \mathrm{~d}$ \\
D & $1.492,63 \pm 1,74 \mathrm{a}$ & $3,63 \pm 0,64 \mathrm{bc}$ & $13,72 \pm 0,11 \mathrm{a}$ & $8,26 \pm 0,18 \mathrm{a}$ & $1137,52 \pm 35,83 \mathrm{a}$ \\
\hline
\end{tabular}

*Médias seguidas por uma mesma letra, na mesma coluna, não diferem significativamente entre si pelo teste de Tukey (p $\leq$
$0,05)$.

Para carotenoides, a amostra A apresentou o teor mais elevado, quando comparado aos demais, no entanto inferior ao reportado por Silva et al., (2013) avaliando polpas de acerola congelada, onde encontraram valores entre de 23,49 mg. $100 \mathrm{~mL}^{-1}$ a $37,04 \mathrm{mg} .100 \mathrm{~mL}^{-1}$ para $\beta$-caroteno, precursor de vitamina $A$. Os resultados do teor de antocianinas para as polpas de acerola encontram-se superiores ao reportado por Gomes (2017) ao avaliar polpas de frutas tropicais para uso em blends (4,30 mg. $\left.100 \mathrm{~g}^{-1}\right)$, exceto a amostra de polpa C.

Quanto ao teor de flavonoides a amostra D apresentou o teor mais elevado, quando comparado com as demais amostras. Mariano-Nasser et al., (2017) avaliando variedades de acerola apresentou valores entre 3,56 a $12,34 \mathrm{mg} .100 \mathrm{~g}^{-1}$, corroborando com os resultados do presente estudo.

Segundo Freire et al., (2013) períodos prolongados de refrigeração ou temperaturas muito baixas geram uma perda na qualidade dos frutos, podendo reduzir o teor de compostos bioativos em seus derivados.
Os compostos fenólicos são os maiores responsáveis pela atividade antioxidante em frutos, observa-se que todas as polpas de acerola apresentaram teores bastante significativos, tornando-se uma fonte potencial desse composto. Vieira et al. (2011) avaliando polpa de acerola obteve teor médio de $835,25 \mathrm{mg} .100 \mathrm{~g}^{-1}$ de ácido gálico, semelhante ao encontrado para a amostra A.

Na Tabela 7, encontram-se os resultados obtidos para as avaliações físico-químicas em polpa de goiaba. Para os sólidos solúveis a legislação exige o valor mínimo de 7,5\% (BRASIL, 2018). Desta forma, somente a amostras B encontram-se dentro dos padrões. Os resultados para $\mathrm{pH}$, somente as amostras B, C e D encontram-se dentro do permitido pela legislação brasileira, na qual exige valor mínimo de 3,5 para polpa de goiaba (BRASIL, 2018). Além disso, todas as amostras obtiveram valores abaixo de 4,5, permitindo uma maior estabilidade quanto ao crescimento de microrganismos.

Tabela 7. Avaliação físico-química em polpa de goiaba de quatro amostras comerciais.

\begin{tabular}{ccccccr}
\hline Amostras & SS (\%) & $\mathrm{pH}$ & $\begin{array}{c}\text { Acidez } \\
\text { Titulável } \\
\text { (\% ác. Cítrico) }\end{array}$ & Ratio & Teor de água (\%) & Cinzas (\%) \\
\hline A & $7,4 \pm 0,10 \mathrm{~b}^{*}$ & $3,39 \pm 0,23 \mathrm{~b}$ & $0,75 \pm 0,04 \mathrm{a}$ & $9,8 \pm 0,35 \mathrm{c}$ & $92,82 \pm 0,68 \mathrm{a}$ & $0,34 \pm 0,02 \mathrm{~b}$ \\
$\mathrm{~B}$ & $7,9 \pm 0,06 \mathrm{a}$ & $3,86 \pm 0,16 \mathrm{a}$ & $0,62 \pm 0,02 \mathrm{~b}$ & $12,7 \pm 0,44 \mathrm{~b}$ & $90,88 \pm 0,24 \mathrm{~b}$ & $0,46 \pm 0,02 \mathrm{a}$ \\
$\mathrm{C}$ & $5,7 \pm 0,00 \mathrm{~d}$ & $3,75 \pm 0,16 \mathrm{ab}$ & $0,71 \pm 0,01 \mathrm{a}$ & $8,0 \pm 0,17 \mathrm{~d}$ & $92,97 \pm 0,08 \mathrm{a}$ & $0,35 \pm 0,01 \mathrm{~b}$ \\
$\mathrm{D}$ & $6,8 \pm 0,10 \mathrm{c}$ & $3,56 \pm 0,11 \mathrm{ab}$ & $0,49 \pm 0,02 \mathrm{c}$ & $13,8 \pm 0,86 \mathrm{a}$ & $91,02 \pm 0,54 \mathrm{~b}$ & $0,36 \pm 0,01 \mathrm{~b}$ \\
\hline
\end{tabular}

*Médias seguidas por uma mesma letra, na mesma coluna, não diferem significativamente entre si pelo teste de Tukey $(\mathrm{p} \leq 0,05)$.

De acordo com os resultados para acidez, as polpas de goiaba, caracterizam-se como acidas, com a amostra A em maior percentual. As quatro amostras avaliadas apresentaram acidez em concordância com a legislação que estabelece valor mínimo 0,4\% (BRASIL, 2018).

Quanto ao ratio, a amostra D obteve o melhor equilíbrio entre sólidos solúveis e ácidos, apresentando uma boa palatabilidade. Com relação aos resultados obtidos para o teor de água das polpas de goiaba, estes apresentaram teores elevados, demonstrando uma maior sensibilidade à deterioração. Para o teor de cinzas, a amostra B se destacou entre as demais, indicando maior quantidade de resíduo inorgânico. 
Na Tabela 8 estão expressos os valores para compostos bioativos em polpas de goiaba. Para ácido ascórbico há uma recomendação mínima de $24 \mathrm{mg} \cdot 100 \mathrm{~g}^{-1}$ segundo a legislação para polpa de goiaba (BRASIL, 2018). Com os resultados obtidos, todas as amostras apresentaram-se abaixo do permitido pela legislação e inferior ao reportado Batista et al. (2013) avaliando polpa de goiaba, onde obtiveram teor médio de $24,39 \mathrm{mg} .100 \mathrm{~g}^{-1}$.

Tabela 8. Avaliação de compostos bioativos em polpa de goiaba de quatro amostras comerciais.

\begin{tabular}{|c|c|c|c|c|c|}
\hline Amostras & $\begin{array}{l}\text { Ácido Ascórbico } \\
\qquad\left(\mathrm{mg} .100 \mathrm{~g}^{-1}\right)\end{array}$ & $\begin{array}{l}\text { Carotenoides } \\
\left(\mu \mathrm{g} .100 \mathrm{~g}^{-1}\right)\end{array}$ & $\begin{array}{l}\text { Antocianinas } \\
\left(\mathrm{mg} .100 \mathrm{~g}^{-1}\right)\end{array}$ & $\begin{array}{l}\text { Flavonoides } \\
\left(\mathrm{mg} .100 \mathrm{~g}^{-1}\right)\end{array}$ & $\begin{array}{c}\text { Compostos fenólicos } \\
\text { (mg.100g }{ }^{-1} \text { de ácido } \\
\text { gálico })\end{array}$ \\
\hline $\mathrm{A}$ & $5,18 \pm 0,85 a^{*}$ & $0,80 \pm 0,07 \mathrm{~b}$ & $0,12 \pm 0,28 b$ & $1,61 \pm 0,28 \mathrm{a}$ & $51,88 \pm 1,15 \mathrm{c}$ \\
\hline B & $5,18 \pm 0,02 \mathrm{a}$ & $0,79 \pm 0,11 b$ & $0,10 \pm 0,32 \mathrm{~b}$ & $1,84 \pm 0,32 \mathrm{a}$ & $72,22 \pm 0,36 \mathrm{a}$ \\
\hline $\mathrm{C}$ & $5,18 \pm 0,85 a$ & $1,51 \pm 0,06 \mathrm{a}$ & $0,37 \pm 0,52 \mathrm{a}$ & $1,76 \pm 0,52 \mathrm{a}$ & $49,57 \pm 0,48 d$ \\
\hline $\mathrm{D}$ & $5,69 \pm 0,59 a$ & $0,79 \pm 0,03 \mathrm{~b}$ & $0,05 \pm 0,29 b$ & $1,76 \pm 0,29 a$ & $63,60 \pm 0,91 b$ \\
\hline
\end{tabular}

*Médias seguidas por uma mesma letra, na mesma coluna, não diferem significativamente entre si pelo teste de Tukey $(\mathrm{p} \leq 0,05)$.

Para carotenoides, a amostra $\mathrm{C}$ apresentou o teor mais elevado, quando comparado aos demais e inferior ao reportado por Venceslau (2013) ao caracterizar goiaba, onde obteve teor médio entre 6,26 a $12,66 \mu \mathrm{g} .100 \mathrm{~g}^{-1}$. O mesmo autor obteve para antocianinas em polpa de goiaba valores entre 0,23 a $0,42 \mathrm{mg} \cdot 100 \mathrm{~g}^{-1}$, somente a amostra C corroborou com os resultados.

Quanto ao teor de flavonoides a amostra B apresentou o teor mais elevado, quando comparado com as demais amostras. Menezes et al., (2016) avaliando polpa de goiaba apresentou teor médio de $11,75 \mathrm{mg} .100 \mathrm{~g}^{-1}$, superior aos resultados do presente estudo. O mesmo autor obteve teor médio de 55,74 mg.100g ${ }^{-1}$ de ácido gálico, corroborando com os resultados do presente estudo. Observa-se que todas as polpas de goiaba apresentaram teores bastante significativos de compostos fenólicos, tornando-se uma fonte potencial desse composto.

\section{CONCLUSÃO}

$\mathrm{O}$ processamento não influencia no aumento do $\mathrm{pH}$ dos doces em massa de goiaba e banana, e das polpas de acerola e goiaba.

Os teores de ácido ascórbico para os doces em massa de goiaba e banana expressam que o processamento adicionado do tratamento térmico influencia no baixo valor deste composto bioativo.

As polpas de acerola apresentam teores consideráveis para o ácido ascórbico e compostos fenólicos, enquanto as polpas de goiabas apresentam baixos teores para todos os compostos bioativos.

\section{REFERÊNCIAS}

ADRIANO, E.; LEONEL, S.; EVENGELISTA, R. M. Qualidade de fruto da aceroleira cv. Olivier em dois estádios de maturação. Revista Brasileira de Fruticultura, v.33, n.1, 2011. 10.1590/S0100-29452011000500073.

AOAC, ASSOCIATION OF OFFICIAL ANALITICAL CHEMISTRS. Official methods of analysis of the association of official analytical chemistrs.18.ed. Gathersburg, 2005. $1015 p$.

BATISTA, A. G.; OLIVEIRA, B. D.; OLIVEIRA, M. A.; GUEDES, T. J.; SILVA, D. F.; PINTOS, N. A. V. D. Parâmetros de qualidade de polpas de frutas congeladas: uma abordagem para produção do agronegócio familiar no Alto
Vale do Jequitinhonha. Revista Tecnologia \& Ciência Agropecuária, v.7, n.4, p.49-54, 2013.

BEZERRA, J. M.; VIEIRA, M. M. S.; SANTOS, A. F.; FARIAS, E. T. R.; LOPES, M. F.; SOUZA, A. S. Composição química de oito cultivares de feijão-caupi. $\begin{array}{lllll}\text { Revista } & \text { Verde, } & \text { v.14, n.1, }\end{array}$ 10.18378/rvads.v14i1.5683.

BRASIL. Agência Nacional de Vigilância Sanitária Resolução $n^{\circ} 269$, de 22 de setembro de 2005. Regulamento Técnico sobre a ingestão Diária Recomendada (IDR) de Proteína, Vitaminas e Minerais. Diário Oficial da União, 2005.

BRASIL. Ministério da Agricultura, Pecuária e Abastecimento. Instrução normativa $n^{\circ} 37$ (continuação), de 8 de outubro de 2018. Parâmetros analíticos e quesitos complementares aos padrões de identidade e qualidade de polpa de fruta.

BRASIL. Resolução Normativa n. 09, de 11 de dezembro de 1978. Aprova as normas técnicas especiais relativas a alimentos e bebidas. Brasília, 1978.

FONSECA, P. A. Q. Análises físico-químicas de polpas de frutas e avaliação dos seus padrões de identidade e qualidade. 2012. 60 f. Dissertação (Mestrado em química), Universidade Federal do Rio Grande do Norte, Natal. 2012.

FRANCIS, F. J. Analysis of anthocyanins. In: MARKAKIS, P. (ed). Anthocyanins as food colors. New York: Academic Press, 1982. p.280.

FREDA, S. A. Doce em massa convencional e light de goiabas (Psidium guajava L.): estabilidade de compostos bioativos, qualidade sensorial e microbiológica. 2014. $99 \mathrm{f}$. Dissertação (Mestrado em Nutrição e Alimentos) Universidade Federal de Pelotas, Pelotas, 2014.

FREDA, S. A., KRUMREICH, F. D., RUTZ, J. K., HARTWIG, N., ZAMBIAZI, R. C. Bioactive compounds during processing and storage of sweet guava (conventional and light). International Food Research Journal, v.25, n.3, p.1181-1188, 2018.

FREIRE, J. M.; ABREU, C. M. P.; ROCHA, D. A.; CORRÊA, A. D.; MARQUES, N. R. Quantificação de 
compostos fenólicos e ácido ascórbico em frutos e polpas congeladas de acerola, caju, goiaba e morango. Ciência Rural, v.43, n.12, 2013. 10.1590/S0103-84782013005000132.

GOMES, J. C.; OLIVEIRA, G. F. Análises físico-químicas de alimentos. Viçosa, MG: Universidade Federal de Viçosa UFV, 2011.

GOMES, J. S. Desenvolvimento de bebida mista a base de frutos tropicais. 2017. 77f. Monografia (Bacharel em Engenharia de Alimentos) - Universidade Federal de Campina Grande. 2017.

HAIDA, K. S.; HAAS, J.; MELLO, S. A.; HAIDA, K. S.; ABRÃO, R. M.; SAHD, R. Compostos fenólicos e atividade antioxidante de goiaba (Psidium guajava L.) fresca e congelada. Revista Fitos, v.9, n.1, p.1-72, 2015. 10.5935/2446-4775.20150004.

INSTITUTO ADOLFO LUTZ, Normas Analíticas métodos químicos e físicos para análise de alimentos. 4 ed. São Paulo: Instituto Adolfo Lutz, 2008.

KATO, T.; RIBEIRO, K. P.; BORDONAL, V. C.; SILVA, M. B. R.; OLIVEIRA, A. F.; SEIBEL. Avaliação da qualidade de doces de frutas agroindustriais do norte do paraná. Revista Brasileira de Produtos Agroindustriais, v.15, n.2, p.173-182, 2013. 10.15871/1517-8595/rbpa.v15n2p173182.

LICHTENTHALER, H. K. Chlorophylls and carotenoids: pigments of photosynthetic biomembranes. In: PACKER, L.; DOUCE, R. (Eds). Methods in Enzimology, v.148, p.350$382,1987$.

LIMA, T. L. S.; CAVALCANTE, C. L.; SOUSA, D. G.; SILVA, P. H. A.; SOBRINHO, L. G. A. Avaliação da composição físico-química de polpas de frutas comercializadas em cinco cidades do Alto Sertão paraibano. Revista Verde, v.10, n.2, p.49-55, 2015. 10.18378/rvads.v10i2.3378.

MARIANO-NASSER, F. A. C.; NASSER, M. D.; FURLANETO, K. A.; RAMOS, J. A.; VIEITES, R. L.; PAGLIARINI, M. K. Compostos bioativos em diferentes cultivares de acerola. Semina: Ciências Agrárias, Londrina, v.38, n.4, p. 2505-2514, 2017. 10.5433/16790359.2017v38n4Sup11p2505.

MARTINS, H. D.; PERFEITO, D. G. A.; SILVA, A. R.; PEIXOTO, N. Caracterização e estudo da estabilidade física de suco misto adoçado de mangaba e cagaita. Revista de Agricultura Neotropical, v.4, n.2, p.81-87, 2017.

MENEZES, P. E.; DORNELLES, L. L.; FOGAÇA, A. O.; BOLIGON, A. A.; ATHAYDE, M. L.; BERTAGNOLLI, S. M. M. Composição centesimal, compostos bioativos, atividade antioxidante e caracterização fenólica da polpa de goiaba. Ciências da Saúde, v.17, n.2, p.205-217, 2016.

MORAIS, E. C.; PATIAS, S. G. O.; FERREIRA, N. S. S.; PICANÇO, N. F. M.; RODRIGUES, E. C.; NASCIMENTO, E.; FARIA, R. A. P. G. Compostos bioativos e características físico-químicas de polpa de araticum in natura e pasteurizada. Braz. J. Food Technol., v. 2 , 2017. 10.1590/19816723.14216 .

OLIVEIRA, E. N. A.; FEITOSA, B. F.; SOUZA, R. L. A. Tecnologia e processamento de frutas: doces, geleias e compotas. Natal: EditoraIFRN, 2018. 316p.

OLIVEIRA, M. M. T.; ALVES, R. E.; SILVA, L. R.; ARAGÃO, F. A. S. Fruit quality of watermelon hybrids with seeds. Rev. Fac. Agron. v.118, n.1, p.77- 83, 2019. 10.24215/16699513e008.

SILVA, F. A. S.; AZEVEDO, C. A. V. The Assistat Software Version 7.7 and its use in the analysis of experimental data. African Journal of Agricultural Research, v.11, n.39, p.37333740, 2016. 10.5897/AJAR2016.11522.

SILVA, L. M. R. Compostos bioativos em polpas e subprodutos de frutas tropicais: quantificação, atividade antimicrobiana e encapsulamento. 2014. 109 f. Tese (Doutorado em Ciências e Tecnologia de Alimentos) Universidade Federal do Ceará, Fortaleza. 2014.

SILVA, M. L. S.; MENEZES, C. C.; PORTELA, J. V. F.; ALENCAR, P. E. B. S.; CARNEIRO, T. B. Teor de carotenoides em polpas de acerola congeladas. Revista Verde, v.8, n.1, p.170-173, 2013.

VENCESLAU, W. C. D. Maturação, conservação e capacidade antioxidante em goiabas "paluma". 2013. $151 \mathrm{f}$. Dissertação (Mestrado em Sistemas Agroindustriais) Universidade Federal de Campina grande. 2013.

VIANA, M. M. S.; CARLOS, L. A.; SILVA, E. C.; PEREIRA, S. M. F.; OLIVEIRA, D. B.; ASSIS, M. L. V. Composição fioquímica e potencial antioxidante em hortaliças não convencionais. Horticultura Brasileira, v. 33, p.504-509, 2015. 10.1590/S0102-053620150000400016.

VIEIRA, L. M.; SOUSA, M. S. B.; MANCINI-FILHO, J.; LIMA, A. Fenólicos totais e capacidade antioxidante in vitro de polpas de frutos tropicais. Revista Brasileira de Fruticultura, v.33, n.3, p.888-897, 2011. 10.1590/S010029452011005000099.

WATERHOUSE, A. Folin-ciocalteau micro method for total phenol in wine. American Journal of Enology and Viticulture, p. 3-5, 2006. 